\title{
Prótese sobre implante dentário parafusada ou cimentada?
}

\author{
Screwed or cemented dental implant prosthesis? \\ ¿Prótesis de implante dental atornillada o cementada?
}

Recebido: 10/11/2021 | Revisado: 19/11/2021 | Aceito: 20/11/2021 | Publicado: 02/12/2021

Graziele Parize

ORCID: https://orcid.org/0000-0002-3463-7865

Universidade Santo Amaro, Brasil

E-mail: grazi.parize@hotmail.com

Taísa Iara Pestana

ORCID: https://orcid.org/0000-0002-6214-1082

Fundação Hermínio Ometto, Brasil

E-mail: taisa_iarap@hotmail.com

Ronald Farhat Cardoso

ORCID: https://orcid.org/0000-0003-2036-4533

Fundação Hermínio Ometto, Brasil

E-mail: fc.ronald@gmail.com

Yeon Jung Kim

ORCID: https://orcid.org/0000-0002-5908-2907

Universidade Santo Amaro, Brasil E-mail: yjkim@prof.unisa.br

Débora Pallos

ORCID: https://orcid.org/0000-0001-7426-1041

Universidade Santo Amaro, Brasil

E-mail: dpallos@prof.unisa.br

\begin{abstract}
Resumo
Os implantes tornaram-se uma alternativa viável no âmbito odontológico devolvendo a função, estética e conforto para o paciente. A escolha do tipo de conexão final da prótese é baseada na situação clínica de cada caso e por preferência do profissional. Existem dois tipos de restaurações protéticas comumente utilizadas na Implantodontia: as próteses cimentadas e parafusadas; ambas com suas vantagens e limitações. O objetivo desse estudo foi de analisar e identificar, através de uma revisão de literatura narrativa, a relação dos diferentes tipos de próteses (cimentada e parafusada) com a perda óssea marginal peri-implantar, assim como, correlacionar o tipo de prótese utilizada com a taxa de sobrevivência do implante. A decisão sobre qual sistema protético usar tem início durante a etapa de planejamento e pode implicar na estética, oclusão, retenção, efeito sobre os tecidos peri-implantares, além de outras complicações, influenciando na taxa de sobrevivência do implante. A perda óssea marginal peri-implantar é um dos problemas relacionados com a falha tardia dos implantes osseointegrados, sendo uma das causas de insucessos, podendo resultar na perda de osseointegração. Sendo assim, conclui-se que, a perda óssea marginal peri-implantar possui etiologia multifatorial; as próteses parafusadas apresentaram mais complicações biomecânicas, como o afrouxamento de parafuso e fratura da cerâmica e as próteses cimentadas apresentaram mais complicações biológicas envolvendo os tecidos peri-implantares.
\end{abstract}

Palavras-chave: Implantes dentários; Prótese dentária fixada por implante; Reabsorção óssea.

\begin{abstract}
Dental implants became a viable alternative involving the Odontology field developing the aesthetics function and the patient's comfort. The choice of which type of prosthesis connection is based on their clinical situation of each case and also for own professional preferences. There are two types of prosthetic restorations commonly used in Implantology: the cemented one and the screw retained prosthesis; both have their own advantages and limitations. The decision of which prosthetic method to use starts during the planning stage and can imply the aesthetics, dental occlusion, retention, effect over the peri-implant tissue, among other complications, influencing the implant survival rate. The peri-implant marginal bone loss is one of the problems related to the late failure of the osseointegration of the implants, being a relevant cause of failure, because it may promote the implant removal due to the osseointegration loss. The goal of this study was to analyze and identify the relation of different types of prosthesis (cemented and screw retained) through a literary point, with the peri-implant marginal bone loss, as well as relate the used type of prosthesis with the implant survival rate. Therefore, it is concluded that the marginal peri-implant bone loss owns multifactorial etiology and there are other factors that influence the implant survival, which is not possible to obtain a straight bone loss comparison with the types of prosthesis installed. Nevertheless, the screw-retained prosthesis presented more biomechanical complications such as the screw loosening and the ceramic fracture; and the cemented prosthesis showed more biological complications involving the peri- implant tissues.
\end{abstract}

Keywords: Dental implants; Dental prosthesis, implant-supported; Bone resorption. 


\begin{abstract}
Resumen
Los implantes se han convertido en una alternativa viable en el campo dental, devolviendo la función, la estética y el confort al paciente. La elección del tipo de conexión final de la prótesis se basa en la situación clínica de cada caso y también en la preferência del profesional. Hay dos tipos de restauraciones protésicas que se utilizan habitualmente en implantología: prótesis cementadas y atornilladas; ambos con sus ventajas y limitaciones. El objetivo de este estudio fue analizar e identificar, a través de una revisión de la literatura, la relación de diferentes tipos de prótesis (cementadas y atornilladas) con la pérdida de hueso marginal periimplantario, así como correlacionar el tipo de prótesis utilizada con la tasa de supervivencia del implante. La decisión sobre qué sistema protésico utilizar comienza durante la etapa de planificación y puede implicar estética, oclusión, retención, efecto sobre los tejidos periimplantarios, además de otras complicaciones, que influyen en la tasa de supervivencia del implante. La pérdida de hueso marginal periimplantario es uno de los problemas relacionados con el fracaso tardío de los implantes osteointegrados, siendo una de las causas del fracaso, que puede resultar en la pérdida de la osteointegración. Por tanto, se concluye que la pérdida de hueso marginal periimplantario tiene una etiología multifactorial; Las prótesis atornilladas tuvieron más complicaciones biomecánicas, como el aflojamiento del tornillo y la fractura de cerámica, y las prótesis cementadas tuvieron más complicaciones biológicas que involucraron los tejidos periimplantarios.
\end{abstract}

Palabras clave: Implantes dentales; Prótesis dental de soporte implantado; Resorción ósea.

\title{
1. Introdução
}

Diante do conceito de osseointegração definido por Bränemark de "uma conexão estrutural direta e funcional entre o osso vital e a superfície de um implante capaz de suportar carga funcional” (Pye et al., 2009; Brügger et al., 2015), a Implantodontia tem avançado e atualmente vem ganhando novas descobertas que são introduzidas no mercado. Alguns procedimentos cirúrgicos como regeneração óssea guiada, além das características dos materiais e a evolução da reabilitação protética tem sido importantes na Odontologia contemporânea (Wittneben, Millen \& Brägger, 2014).

Existem diferentes filosofias a respeito do tipo de conexão final utilizada na reabilitação com próteses sobre implante, sendo elas: a parafusada ou parafuso-retida e a cimentada ou cimento-retida. Em geral, essa escolha baseia-se no tipo de situação clínica encontrada e também na preferência do profissional por um sistema ou outro (Chee \& Jivraj, 2006; Lee, Okayasu \& Wang, 2010; Nissan et al. 2011; Sherif et al., 2011; Sailer et al., 2012; Shadid \& Sadaqa, 2012; Lee et al., 2013;Wittneben, Millen \& Brägger, 2014; Koller, Pereira-Cenci \& Boscato, 2016;Lemos et al., 2016; Francis, Pillai \& Lylajam,2018; Hamed et al., 2020).

A literatura nos mostra vantagens e desvantagens em relação ao uso de cada tipo de conexão protética (Chee \& Jivraj, 2006; Lee, Okayasu \& Wang, 2010; Shadid \& Sadaqa, 2012; Francis, Pillai \& Lylajam, 2018; Gómez-Polo et al., 2018). O sistema com prótese cimentada se difere da parafusada principalmente em relação ao tipo de retenção, já que as cimentadas são através de cimentos provisórios ou definitivos e as parafusadas apenas com retenção mecânica do parafuso no implante ou intermediário protético (Chee \& Jivraj, 2006; Lee, Okayasu \& Wang, 2010; Sherif et al., 2011; Shadid \& Sadaqa, 2012; Wittneben, Millen \& Brägger, 2014). Além disso, as próteses parafusadas se sobressaem quanto á recuperabilidade, que para fins de manutenção pode ser removida e seus componentes substituídos; contudo, quando ocorrem complicações nas próteses cimentadas, geralmente toda a peça tem que ser reconfeccionada (Lee, Okayasu \& Wang, 2010; Shadid \& Sadaqa, 2012; Wittneben, Millen \& Brägger, 2014; Lemos et al., 2016).

Apesar de serem relatados resultados promissores com as próteses sobre implante, as complicações e falhas podem ocorrer e a literatura tem mostrado morbidades associadas ao insucesso do tratamento com implantes dentários (Sailer et al., 2012; Shadid \& Sadaqa, 2012; De Brandão, Vettore \& Vidigal Junior, 2013; Wittneben, Millen \& Brägger, 2014; Lemos et al., 2016). Desse modo, os tecidos peri-implantares podem ser susceptíveis às condições de doença ou complicações (Pjetursson et al., 2004; Lee, Okayasu \& Wang, 2010; Shadid \& Sadaqa, 2012; De Brandão, Vettore \& Vidigal Junior, 2013; Wittneben, Millen \& Brägger, 2014; Lemos et al., 2016).

A partir dessa análise, o insucesso ou a falha de um implante pode ser determinado através de parâmetros e medições quantitativas e qualitativas (Albrektsson et al., 1986; Sakka, Baroudi \& Nassani, 2012; Brügger et al., 2015) que os definem 
como falha precoce, após a fase cirúrgica, quando não ocorre a osseointegração (Sakka, Baroudi \& Nassani, 2012; Brügger et al., 2015), ou falha tardia, após a osseointegração e instalação protética (Esposito et al., 1999; Di Iorio et al., 2011; Linkevicius et al., 2013). Sendo assim, as doenças peri-implantares (Pye et al., 2009; Linkevicius et al., 2013; Romanos et al., 2015) e os problemas biomecânicos derivados das próteses instaladas, são as causas mais frequentes das falhas tardias dos implantes relacionadas à perda óssea marginal peri- implantar (Di Iorio et al., 2011; Firme et al., 2014; Koller, Pereira-Cenci \& Boscato, 2016). Diante do exposto, o presente trabalho irá apresentar a relação das próteses cimentadas e parafusadas com a perda óssea marginal peri-implantar, bem como verificar se um tipo de conexão protética é mais suscetível à perda óssea em relação a outro.

O objetivo principal desse estudo foi de analisar e identificar, através de uma revisão de literatura, a relação das próteses cimentadas e parafusadas com a perda óssea marginal peri-implantar.

\section{Metodologia}

O presente estudo trata-se de uma revisão narrativa, comparando as indicações e contraindicações de próteses sobre implantes cimentadas e parafusadas. A revisão abrangeu artigos científicos disponíveis nas bases de dados: Pubmed (National Libary of Medicine), Scielo (Scientific Eletronic Library Online). Descartou-se estudos que não apresentavam o resumo, e não abordavam a temática do estudo. Este levantamento foi realizado no período de 2000 a 2021. Foram inclusos artigos em inglês, espanhol ou português, sob a forma de artigo completo, revisões sistemáticas, relatos de casos, ensaios clínicos randomizados, estudos clínicos transversais ou de coorte. Foram excluídos os artigos que não se adequavam aos critérios de inclusão.

\section{Revisão da Literatura}

$\mathrm{Na}$ literatura, vários fatores têm sido propostos para explicar as alterações ósseas peri-implantares e um importante critério utilizado para determinar a taxa de sucesso ou falha de um implante é a perda óssea marginal (Albrektsson et al., 1986; Jung et al., 2008; Sailer et al., 2012; Vigolo et al., 2012; Koller, Pereira-Cenci \& Boscato, 2016).

Em próteses sobre implante, a união da estrutura protética ao pilar pode ocorrer por meio do aparafusamento ou cimentação (Chee \& Jivraj, 2006; Sailer et al., 2012; Shadid \& Sadaqa, 2012; Francis, Pillai \& Lylajam, 2018). A escolha do tipo de conexão final da prótese é baseada na situação clínica de cada caso, tendo início na etapa de planejamento, como também, pode ser eleita pela preferência do profissional (Lee, Okayasu \& Wang, 2010; Sailer et al., 2012; Shadid \& Sadaqa, 2012; Vigolo et al., 2012; Lemos et al., 2016; Gómez-Polo et al., 2018; Hamed et al., 2020). Contudo, a decisão sobre qual sistema protético usar pode implicar em resultados não satisfatórios e levar a complicações protéticas e biológicas (Jung et al., 2008; Lee, Okayasu \& Wang, 2010; Di Iorio et al., 2011; Shadid \& Sadaqa, 2012; De Brandão, Vettore \& Vidigal Junior, 2013; Wittneben, Millen \& Brägger, 2014; Ferreiroa et al., 2015; Lemos et al., 2016; Hamed et al., 2020).

Para melhor entendimento das circunstâncias que podem levar à perda óssea marginal peri-implantar em próteses parafusadas e cimentadas, nesse estudo serão expostas algumas diferenças e consequências no uso de um sistema ou outro, associada à falha tardia dos implantes.

\section{Estética}

Em geral, quando um implante é instalado na posição ideal, partindo do planejamento prévio adequado, a estética previsível pode ser alcançada tanto com a conexão final parafusada ou cimentada (Chee \& Jivraj, 2006; Shadid \& Sadaqa, 2012; Francis, Pillai \& Lylajam, 2018; Hamed et al., 2020). Porém, se o implante estiver em uma posição cujo acesso do parafuso saia na área estética é indicado optar por uma prótese cimentada. Em outros casos, onde a posição está desfavorável, 
são utilizados pilares pré-fabricados angulados ou personalizados pelo profissional, sendo redirecionados para o cíngulo ou superfície oclusal (Chee \& Jivraj, 2006; Lee, Okayasu \& Wang, 2010; Shadid \& Sadaqa, 2012; Gómez-Polo et al., 2018). Mesmo em regiões satisfatórias, o acesso ao parafuso do implante pode ser mascarado pelo uso de resinas opacas que oferecem uma melhora significativa na estética destas restaurações (Lee, Okayasu \& Wang, 2010; Shadid \& Sadaqa, 2012; Vigolo et al., 2012).

\section{Retenção e Manutenção (Recuperabilidade)}

Várias propriedades afetam a retenção de uma prótese, sendo a área de superfície de contato, altura da superfície, além do tipo de cimento utilizado, como no caso da prótese cimentada. A maioria dos pilares fabricados é feito com base no conceito de cone, com aproximadamente 6 graus de inclinação, assim como é proposto no preparo dos dentes naturais (Shadid \& Sadaqa, 2012). Em relação à altura do pilar, estes geralmente estão localizados de $2 \mathrm{~mm}$ a $3 \mathrm{~mm}$ subgengivalmente da margem da coroa ao implante, o que aumenta a superfície de retenção quando comparado a dentes. Já quando a altura protética for limitada $(4 \mathrm{~mm})$, a prótese parafusada é indicada, podendo ser fixada diretamente no implante sem o pilar intermediário (Lee, Okayasu \& Wang, 2010; Shadid \& Sadaqa, 2012; Gómez-Polo et al., 2018; Hamed et al., 2020). Além disso, o tipo de cimento escolhido é um fator de grande influência na prótese cimentada, podendo ser utilizado o método de cimentação progressiva até obter a retenção desejada (Lee, Okayasu \& Wang, 2010; Vigolo et al., 2012).

Uma grande vantagem da prótese parafusada é que ela permite a remoção dos componentes para manutenção sem danos na sua estrutura, principalmente em caso de próteses múltiplas e unidas, assim, é possível remover a restauração do acesso do parafuso, remover a conexão protética e realizar a manutenção dos componentes, aperto do parafuso e a limpeza dos tecidos moles (Lee, Okayasu \& Wang, 2010; Sailer Et Al., 2012; Shadid \& Sadaqa, 2012; Vigolo Et Al., 2012; Gaddale, Mishra \& Chowdhary, 2020). Quanto à prótese cimentada, nesta pode ser utilizado cimentos provisórios a fim de permitir uma manutenção futura, já que em alguns casos não é possível recuperar uma prótese cimentada após danos na estrutura ou componente, sendo necessária a confecção de uma nova. (Lee, Okayasu \& Wang, 2010; Sailer et al., 2012; Shadid \& Sadaqa, 2012; Vigolo et al., 2012).

\section{Oclusão}

As próteses cimentadas não apresentam orifício de acesso na superfície oclusal, com isso, a carga axial pode ser direcionada para o implante. Nas próteses parafusadas, quanto maior a plataforma utilizada, maior o orifício de acesso ao parafuso e maior o tamanho da restauração que será realizada, podendo ocupar cerca de 50\% a 75\% da oclusal nos posteriores, além de comprometer a durabilidade em longo prazo, podem interferir não só com os contatos oclusais cêntricos, como também com os movimentos excêntricos de lateralidade e protrusão (Shadid \& Sadaqa, 2012).

Os contatos laterotrusivos, tanto no guia canino quanto na função de grupo, precisam fornecer um guia adequado para desocluir simultaneamente todos os implantes do lado de não trabalho. Da mesma forma, na posição protrusiva também deve desocluir todos os implantes posteriores (Koller, Pereira-Cenci \& Boscato, 2016). Diante disso, é necessário realizar um correto ajuste oclusal após a instalação das próteses (Lee, Okayasu \& Wang, 2010; Vigolo et al., 2012; Koller, Pereira-Cenci \& Boscato, 2016; Radaelli et al., 2020).

\section{Complicações Biomecânicas}

A falta de passividade principalmente em próteses múltiplas tem sido correlacionada como um fator para as complicações protéticas. A passividade na prótese é desejada, pois ajuda a diminuir tensões que são aplicadas para o osso e pilar do implante. Em uma prótese onde a conexão não é passiva, ou seja, seu encaixe é forçado, as tensões são direcionadas à 
supraestrutura da prótese, ao osso e implante, o que pode levar à perda óssea, fratura do componente e/ou implante, ou mesmo mobilidade. Contudo, quando um implante é instalado paralelo às cargas oclusais, a distribuição das forças é mais favorável (Lee, Okayasu \& Wang, 2010; Di Iorio et al., 2011; Lee et al., 2013; Firme et al., 2014).

Outro fator que pode interferir na passividade é uma moldagem inadequada, que pode causar desvios na carga vertical do implante, levando ao afrouxamento ou fratura do parafuso (Sailer et al., 2012; Lee et al., 2013). Uma estrutura não passiva aumenta a probabilidade de afrouxamento do parafuso quando em função (Jung et al., 2008; Di Iorio et al., 2011; Nissan et al., 2011; Sailer et al., 2012; Silva et al., 2014; Wittneben, Millen \& Brägger, 2014; Ferreiroa et al., 2015; Gaddale, Mishra \& Chowdhary, 2020; Hamed et al., 2020). Isso pode variar de 10\% a 65\% em alguns estudos, geralmente ocorre na região de molar e áreas de cantlever (Lee, Okayasu \& Wang, 2010; Wittneben, Millen \& Brägger, 2014).

Por outro lado, um ajuste passivo absoluto pode ser difícil de conseguir com os procedimentos convencionais de fundição, já que ele é afetado por cada etapa do processo de fabricação da prótese (Lee et al., 2013; Firme et al., 2014). É interessante notar que a maioria das próteses não apresenta um ajuste completamente passivo e mesmo assim é funcional, sugerindo certa tolerância biológica ao desajuste (Lee, Okayasu \& Wang, 2010). Entretanto, alguns métodos assistidos por computador são capazes de confeccionar estruturas com maior precisão em relação aos métodos tradicionais (Sailer et al., 2012; Lee et al., 2013; Firme et al., 2014).

O desajuste da prótese implanto-suportada pode causar tensão indesejável, incluindo carga prejudicial ao osso, que consequentemente pode levar ao desenvolvimento de uma microflora na interface pilar-implante e resultar em inflamação crônica causando alterações na região (Piattelli et al., 2001; Lee, Okayasu \& Wang, 2010; Sailer et al., 2012; Lee et al., 2013).

Na prótese parafusada, o ideal é que não haja espaço entre a coroa e o pilar; e na prótese cimentada, o pilar é parafusado ao implante e o espaço para o cimento ajuda a conferir a passividade, atuando como um amortecedor e reduzindo as tensões no osso (Lee, Okayasu \& Wang, 2010; Shadid \& Sadaqa, 2012; Lee et al., 2013; Hamed et al., 2020).

A fratura da porcelana também é um fator das complicações protéticas e mais suscetível de ser observada em próteses parafusadas (Lee, Okayasu \& Wang, 2010; Nissan et al., 2011; Sailer et al., 2012; Shadid \& Sadaqa, 2012; Vigolo et al., 2012; Wittneben, Millen \& Brägger, 2014; Ferreiroa et al., 2015; Lemos et al., 2016). Os materiais restauradores do acesso ao parafuso são propensos ao desgaste sob forças funcionais e assim, os contatos oclusais são menos preservados (Nissan et al., 2011; Shadid \& Sadaqa, 2012; LEMOS et al., 2016). Diante do fato de que os implantes não possuem ligamento periodontal, as forças oclusais são aplicadas na região do acesso do parafuso, o que afeta a direção das cargas oclusais que serão distribuídas como forças laterais ao implante em vez de serem direcionadas axialmente, tendo como consequência a ocorrência de trincas na peça e levar a fratura da porcelana (Lee, Okayasu \& Wang, 2010; Di Iorio et al., 2011; Sailer et al., 2012; Vigolo et al., 2012; Wittneben, Millen \& Brägger, 2014).

Alguns estudos relataram que guias oclusais inadequados produziam forças desfavoráveis nas estruturas de suporte e maior concentração de estresse no contato osso-implante (Sherif et al., 2011; Vigolo et al., 2012; Koller, Pereira-Cenci \& Boscato, 2016). Um estudo revelou que o guia lateral envolvendo dente e implante obteve maior perda óssea ao redor dos implantes do que o guia lateral canino envolvendo apenas dentes ou implantes. Provavelmente, isso ocorreu porque existem diferenças na intensidade das forças aplicadas ao redor do dente e do implante, uma vez que os implantes não possuem um feedback periodontal com mecanorreceptores. Em razão disso, o controle motor da mastigação é reduzido e não restringe a carga aplicada às próteses implanto-suportadas, resultando em maior estresse no contato osso e implante, do que no contato osso e dente (Koller, Pereira-Cenci \& Boscato, 2016).

Posto isso, todas as forças prejudiciais, fora do eixo, incluindo os contatos oclusais excessivos e cantilevers, devem ser avaliados e completamente eliminados se possível (Lee, Okayasu \& Wang, 2010; Shadid \& Sadaqa, 2012; Vigolo et al., 2012; Lee et al., 2013; Silva et al., 2014; Koller, Pereira-Cenci \& Boscato, 2016; Radaelli et al., 2020). 


\section{Complicações dos Tecidos Peri-Implantares}

O cimento residual nas regiões de margem da prótese, margem gengival, sulco gengival ou área subgengival; é uma complicação comum em próteses cimentadas e envolve os tecidos peri-implantares (Lee, Okayasu \& Wang, 2010; Linkevicius et al., 2011; Sailer et al., 2012; Wadhwani et al., 2012; De Brandão, Vettore \& Vidigal Junior, 2013; Linkevicius et al., 2013; Wittneben, Millen \& Brägger, 2014; Ferreiroa et al., 2015; Gaddale, Mishra \& Chowdhary, 2020; Hamed et al., 2020). Um estudo in vitro mostrou que em todas as amostras testadas havia resíduo de cimento presente, independentemente da posição da coroa em relação à margem gengival (Linkevicius et al., 2011).

A respeito do uso de cimento na prótese cimentada, a técnica tem a desvantagem de permitir o extravasamento de resíduos de cimento para a região subgengival, logo, pode causar inflamação peri-implantar associada ao inchaço, dor, aumento da profundidade de sondagem, sangramento ou exsudação na sondagem, o que pode levar à perda óssea marginal peri-implantar (Lee, Okayasu \& Wang, 2010; Shadid \& Sadaqa, 2012; Wadhwani et al., 2012;De Brandão, Vettore \& Vidigal Junior, 2013; Linkevicius et al., 2013; Koller, Pereira-Cenci \& Boscato, 2016; Gaddale, Mishra \& Chowdhary, 2020). Além disso, alguns estudos mostraram uma forte relação entre os resíduos do cimento e o desenvolvimento de doenças periimplantares (Linkevicius et al., 2011; Wadhwani et al., 2012; Linkevicius et al., 2013; Ferreiro et al., 2015).

As doenças peri-implantares consistem em mucosite peri-implantar e pela peri-implantite, resultando em lesões inflamatórias que surgem em decorrência de um desequilíbrio entre os componentes microbiológicos e a resposta do hospedeiro, envolvendo os tecidos moles e duros circundantes ao implante em função. A mucosite peri-implantar é definida como uma lesão inflamatória reversível que envolve apenas os tecidos moles peri- implantares. A peri-implantite afeta tanto os tecidos moles quanto os tecidos duros e é caracterizada pela perda de suporte ósseo em torno do implante (Pye et al., 2009; Mombelli \& Décaillet, 2011; Romanos et al., 2015). A falta ou má higiene bucal é considerada um fator determinante para o desenvolvimento das doenças peri-implantares, visto que, o acúmulo de placa pode causar a inflamação dos tecidos (Pye et al., 2009; Mombelli \& Décaillet, 2011; Linkevicius et al., 2013).

Alguns autores relatam que a prótese implanto-suportada cimentada apresenta maior tendência ao acúmulo de placa, sangramento sulcular e inflamação gengival (Sherif et al., 2011; Sailer et al., 2012; Ferreiroa et al., 2015; Koller, Pereira-Cenci $\&$ Boscato, 2016).

Para prevenir esse tipo de complicação é crucial remover todo o excesso do cimento na instalação da prótese, seja ela provisória ou não (Sailer et al.,2012; Vigolo et al., 2012; Wadhwani et al., 2012; Linkevicius et al., 2013).

Algumas técnicas podem ser utilizadas, como colocar um pouco de vaselina na parte externa da superfície da coroa, remover cuidadosamente com uma cureta o excesso do cimento e usar o fio dental (Shadid \& Sadaqa, 2012).

\section{Discussão}

Visto que, os clínicos consideram as vantagens e limitações de cada tipo de conexão para cada paciente ao escolher um sistema de retenção ideal para uso, a escolha deve basear-se em evidências para melhorar a longevidade da prótese e implante. Lee, Okayasu e Wang (2010), Shadid e Sadaqa (2012) e Hamed et al. (2020), relataram as características das próteses cimentadas e parafusadas, assim como sua influência na estética, retenção, oclusão, acesso, efeitos na saúde dos tecidos peri-implantares e complicações frequentes.

De Brandão et al. (2013) compararam a perda óssea marginal peri-implantar em uma revisão sistemática entre as próteses parafusadas e cimentadas, contudo, apenas dois dos estudos incluídos avaliavam os dois tipos de retenção diretamente e os outros foram comparados indiretamente. Com isso, a perda óssea marginal média combinada foi maior para o grupo das próteses parafusadas. Estes resultados confirmam a mesma assertiva de Nissan et al. (2011), que dentro das complicações biomecânicas, a perda óssea marginal foi mais evidente nas próteses parafusadas. 
De acordo com o exposto por Ferreiro et al. (2015), as próteses parafusadas apresentaram maiores complicações mecânicas, como o afrouxamento do parafuso; já as próteses cimentadas, apresentaram mais complicações biológicas, como mucosite peri-implantar e em alguns casos peri-implantite. Por outro lado, estudos apresentados anteriormente por Sailer et al. (2012), apontam que as próteses parafusadas também estão associadas às complicações biológicas.

Lemos et al., (2016) compararam os sistemas de retenção das próteses cimentadas e parafusadas em relação às complicações protéticas, perda óssea marginal e sobrevivência do implante. Concluíram que, a taxa de complicações protéticas foi estatisticamente maior para as próteses parafusadas, assim como, apresentaram uma diferença significativa para a perda óssea marginal. Assim, a taxa de sobrevivência do implante foi maior nos casos em que instalaram próteses cimentadas.

Dentre as complicações apresentadas por Lemos et al. (2016), a mais frequente foi o afrouxamento do parafuso, concordando com estudos de Sailer et al. (2012) e Shadid e Sadaqa (2012) que demonstraram que a prótese parafusada apresenta mais complicações técnicas do que a cimentada. Essa frequência pode ser explicada devido à maior pré-carga do ajuste passivo reduzido da estrutura das próteses parafusadas. Embora o afrouxamento do parafuso seja um problema mais simples de ser resolvido nas próteses parafusadas, o mesmo geralmente não ocorre com as próteses cimentadas e o acesso a ele pode levar a um contratempo para o clínico.

Contudo, Nissan et al. (2011) que comparava os resultados a longo prazo e as complicações dos dois tipos de conexão protética, mostrou que além do afrouxamento do parafuso, a fratura da cerâmica também foi uma das complicações mais encontradas, sendo a maioria presente nas conexões parafusadas; o que foi confirmado depois no estudo de Wittneben, Millen e Brägger (2014). Além disso, Nissan et al. (2011) revelou que o afrouxamento do parafuso ocorreu em $86 \%$ dos casos verificados de fratura da cerâmica.

Recentemente, Gaddale, Mishra e Chowdhary (2020), avaliaram as complicações técnicas e biológicas de próteses de arcada completa parafusada e cimentadas. Observaram que em geral, as próteses parafusadas apresentaram mais complicações em relação à cimentada. Dentre as complicações técnicas, o afrouxamento e fratura do parafuso ocorreram mais nas próteses parafusadas. E as complicações biológicas, como perda óssea marginal $>2 \mathrm{~mm}$, ocorreram com mais frequência nas próteses cimentadas.

A perda óssea marginal peri-implantar foi avaliada por Firme et al. (2014) que englobou as próteses fixas unitárias e múltiplas sobre implante. Observaram que as próteses parafusadas múltiplas tendem a apresentar maior perda óssea em relação às próteses unitárias, o que pode ser uma consequência da dificuldade em obter um ajuste passivo para todas as regiões da prótese. Entretanto, o estudo não incluiu a relação das próteses cimentadas múltiplas para análise direta, sugerindo um controle dos resultados e assim, não foi possível obter uma diferença significativa entre elas.

Francis, Pillai e Lylajam (2018) avaliaram e compararam as complicações biológicas e protéticas entre as próteses parafusadas e cimentadas, ocorridas no período de 6 meses de carga funcional. Fizeram um modelo de estudo de boca dividida em 14 participantes com ausência dos primeiros molares inferiores (36 e 46). De um lado, instalaram uma prótese parafusada (grupo I) e do outro, uma prótese cimentada (grupo II). Após o período de acompanhamento, concluíram que nenhum dos modelos obteve diferença estatística significante em relação à perda óssea marginal, índice de placa, profundidade de sondagem e afrouxamento do parafuso. Entretanto, em todos os casos, os implantes foram instalados perpendicularmente a oclusão e os participantes mantiveram um nível de higiene alto.

Koller, Pereira-Cenci e Boscato (2016) avaliaram retrospectivamente a associação entre os parâmetros oclusais, periodontais e relacionados ao implante e prótese, com a perda óssea marginal peri-implantar. Como resultado, o estudo mostrou que em implantes envolvidos em esquema oclusal inadequado (ausência de guias e contato oclusal no lado de não trabalho, por exemplo), apresentaram maior perda óssea. Além disso, a presença de placa visível e próteses cimentadas também foram associadas à maior perda óssea peri- implantar. 
Estudos realizados por Sherif et al. (2011) e Linkevicius et al. (2013), mostraram que as próteses cimentadas sobre implante eram mais propensas a apresentarem acúmulo de placa e inflamação gengival, devido à dificuldade inerente em remover o excesso de cimento residual subgengival, pois o acúmulo de placa pode aumentar os riscos de inflamação e, consequentemente, levar à perda óssea marginal.

Em relação às complicações biológicas dos tecidos peri-implantares, Nissan et al. (2011) mostrou que 9,7\% das coroas tiveram complicações leves nos tecidos ou peri-implantite e que $6,3 \%$ incluíram perda óssea $\geq 2 \mathrm{~mm}$ no período de acompanhamento de cinco anos. Posteriormente, Wittneben, Millen \& Brägger (2014) em seus estudos, confirmaram que além destas complicações, a presença de mucosite peri-implantar, fístula ou exsudato e retração, também foram observadas nos dois sistemas protéticos.

Mombelli e Décaillet (2011) realizaram uma revisão descrevendo a microbiota associada à doença peri-implantar e utilizaram 29 relatórios sobre o tema. Constataram em seus resultados que a maioria das amostras analisadas apresentou uma microbiota anaeróbia mista, além disso, também relataram que o infiltrado inflamatório em decorrência do biofilme foi encontrado na mesma proporção em dentes naturais e em implantes, correlacionando os microorganismos presentes na doença periodontal com os presentes na doença peri-implantar.

Linkevicius et al. (2011) avaliaram a quantidade de excesso do cimento residual após a cimentação e a limpeza dessas próteses cimentadas com diferentes níveis de margem gengival. Identificaram que em todos os modelos que variavam de $1 \mathrm{~mm}$ supragengival a 3mm subgengival, havia excesso de cimento não removido, concluindo que, quanto mais subgengival estiver, maior o acúmulo de cimento residual e maior a dificuldade em detectar e removê-lo. Nesse e em outro estudo realizado mais tarde por Linkevicius et al. (2013), demonstraram que o cimento residual está associado ao desenvolvimento das doenças periimplantares, como fator predisponente para o surgimento dessas complicações.

Em geral, autores como de Brandão, Vettore e Vidigal Jr. (2013) e Firme et al. (2014), mencionaram que a perda óssea marginal tem etiologia multifatorial e por isso alguns fatores como a posição do implante, dimensão da plataforma, largura biológica em torno do implante, modelo do implante e do pilar utilizado, presença ou não de cimento residual submucoso, além de outros fatores que não foram controlados e avaliados em outros estudos, devem ser considerados em ensaios clínicos futuros a fim de diminuir o risco de alteração no desfecho destes estudos e obter maior precisão dos riscos que podem levar à perda óssea marginal peri-implantar.

\section{Considerações Finais}

A perda óssea marginal peri-implantar possui etiologia multifatorial e fatores como a falta de passividade, sobrecarga, má higiene, acúmulo de biofilme, falta de ajuste oclusal, entre outros fatores relacionados às próteses instaladas, influenciam diretamente a sobrevivência do implante. As próteses parafusadas sobre implante apresentam mais complicações biomecânicas, como o afrouxamento do parafuso e fratura da cerâmica. Já as próteses cimentadas sobre implante apresentam mais complicações biológicas envolvendo os tecidos peri-implantares.

O excesso de cimento residual das próteses cimentadas sobre implante atua como fator predisponente para o desenvolvimento das doenças peri- implantares (mucosite peri-implantar e peri-implantite). Não há nenhum consenso até o momento sobre qual sistema de retenção é superior a outro para evitar a perda óssea marginal peri-implantar. Assim, a escolha do uso da prótese cimentada ou parafusada deve ser tomada não só pela preferência do profissional, como também, basear-se em evidências científicas e clínicas. 


\section{Referências}

Albrektsson, T., Zarb, G., Worthington, P., \& Eriksson, A. R. (1986). The long-term efficacy of currently used dental implants: a review and proposed criteria of success. The International journal of oral \& maxillofacial implants, 1(1), 11-25.

Brügger, O. E., Bornstein, M. M., Kuchler, U., Janner, S. F., Chappuis, V., \& Buser, D. (2015). Implant therapy in a surgical specialty clinic: an analysis of patients, indications, surgical procedures, risk factors, and early failures. The International journal of oral \& maxillofacial implants, 30(1), 151-160. https://doi.org/10.11607/jomi.3769

de Brandão, M. L., Vettore, M. V., \& Vidigal Júnior, G. M. (2013). Peri-implant bone loss in cement- and screw-retained prostheses: systematic review and meta-analysis. Journal of clinical periodontology, 40(3), 287-295. https://doi.org/10.1111/jcpe.12041

Del'Acqua, M. A., Chávez, A. M., Compagnoni, M. A., \& Molo, F., Jr (2010). Accuracy of impression techniques for an implant-supported prosthesis. The International journal of oral \& maxillofacial implants, 25(4), 715-721.

Di Iorio, D., Sinjari, B., Feragalli, B., \& Murmura, G. (2011). Biomechanical aspects in late implant failures: scanning electron microscopy analysis of four clinical cases. The journal of contemporary dental practice, 12(5), 356-360. https://doi.org/10.5005/jp-journals-10024-1059

Esposito, M., Hirsch, J., Lekholm, U., \& Thomsen, P. (1999). Differential diagnosis and treatment strategies for biologic complications and failing oral implants: a review of the literature. The International journal of oral \& maxillofacial implants, 14(4), 473-490.

Ferreiroa, A., Peñarrocha-Diago, M., Pradíes, G., Sola-Ruiz, M. F., \& Agustín-Panadero, R. (2015). Cemented and screw-retained implant-supported singletooth restorations in the molar mandibular region: A retrospective comparison study after an observation period of 1 to 4 years. Journal of clinical and experimental dentistry, 7(1), e89-e94. https://doi.org/10.4317/jced.51708

Firme, C. T., Vettore, M. V., Melo, M., \& Vidigal, G. M., Jr (2014). Peri-implant bone loss around single and multiple prostheses: systematic review and meta-analysis. The International journal of oral \& maxillofacial implants, 29(1), 79-87. https://doi.org/10.11607/jomi.3316

Francis, L.; Pillai, S.B.; Lylajam, S. (2018) Clinical and radiological evaluation of screw-retained and cement-retained single-implant restorations - A comparative study. International journal of oral care and research, 6 (2),60-66.

Gaddale, R., Mishra, S. K., \& Chowdhary, R. (2020). Complications of screw- and cement-retained implant-supported full-arch restorations: a systematic review and meta-analysis. International journal of oral implantology (Berlin, Germany), 13(1), 11-40.

Gómez-Polo, M., Ortega, R., Gómez-Polo, C., Celemin, A., \& Del Rio Highsmith, J. (2018). Factors Affecting the Decision to Use Cemented or ScrewRetained Fixed Implant-Supported Prostheses: A Critical Review. The International journal of prosthodontics, 31(1), 43-54. https://doi.org/10.11607/ijp.5279

Hamed, M. T., Abdullah Mously, H., Khalid Alamoudi, S., Hossam Hashem, A. B., \& Hussein Naguib, G. (2020). A Systematic Review of Screw versus Cement-Retained Fixed Implant Supported Reconstructions. Clinical, cosmetic and investigational dentistry, 12, 9-16. https://doi.org/10.2147/CCIDE.S231070

Jung, R. E., Pjetursson, B. E., Glauser, R., Zembic, A., Zwahlen, M., \& Lang, N. P. (2008). A systematic review of the 5-year survival and complication rates of implant-supported single crowns. Clinical oral implants research, 19(2), 119-130. https://doi.org/10.1111/j.1600-0501.2007.01453.X

Koller, C. D., Pereira-Cenci, T., \& Boscato, N. (2016). Parameters Associated with Marginal Bone Loss around Implant after Prosthetic Loading. Brazilian dental journal, 27(3), 292-297. https://doi.org/10.1590/0103-6440201600874

Lee, A., Okayasu, K., \& Wang, H. L. (2010). Screw- versus cement-retained implant restorations: current concepts. Implant dentistry, 19(1), 8-15. https://doi.org/10.1097/ID.0b013e3181bb9033

Lee, M. Y., Heo, S. J., Park, E. J., \& Park, J. M. (2013). Comparative study on stress distribution around internal tapered connection implants according to fit of cement- and screw-retained prostheses. The journal of advanced prosthodontics, 5(3), 312-318. https://doi.org/10.4047/jap.2013.5.3.312

Lemos, C. A., de Souza Batista, V. E., Almeida, D. A., Santiago Júnior, J. F., Verri, F. R., \& Pellizzer, E. P. (2016). Evaluation of cement-retained versus screw-retained implant-supported restorations for marginal bone loss: A systematic review and meta-analysis. The Journal of prosthetic dentistry, 115(4), 419-427. https://doi.org/10.1016/j.prosdent.2015.08.026

Linkevicius, T., Puisys, A., Vindasiute, E., Linkeviciene, L., \& Apse, P. (2013). Does residual cement around implant-supported restorations cause periimplant disease? A retrospective case analysis. Clinical oral implants research, 24(11), 1179-1184. https://doi.org/10.1111/j.1600-0501.2012.02570.X

Linkevicius, T., Vindasiute, E., Puisys, A., \& Peciuliene, V. (2011). The influence of margin location on the amount of undetected cement excess after delivery of cement-retained implant restorations. Clinical oral implants research, 22(12), 1379-1384. https://doi.org/10.1111/j.1600-0501.2010.02119.x

Mombelli, A., \& Décaillet, F. (2011). The characteristics of biofilms in peri-implant disease. Journal of clinical periodontology, 38 Suppl 11, 203-213. https://doi.org/10.1111/j.1600-051X.2010.01666.x

Nissan, J., Narobai, D., Gross, O., Ghelfan, O., \& Chaushu, G. (2011). Long-term outcome of cemented versus screw-retained implant-supported partial restorations. The International journal of oral \& maxillofacial implants, 26(5), 1102-1107.

Piattelli, A., Scarano, A., Paolantonio, M., Assenza, B., Leghissa, G. C., Di Bonaventura, G., Catamo, G., \& Piccolomini, R. (2001). Fluids and microbial penetration in the internal part of cement-retained versus screw-retained implant-abutment connections. Journal of periodontology, 72(9), 1146-1150. https://doi.org/10.1902/jop.2000.72.9.1146

Pjetursson, B. E., Tan, K., Lang, N. P., Brägger, U., Egger, M., \& Zwahlen, M. (2004). A systematic review of the survival and complication rates of fixed partial dentures (FPDs) after an observation period of at least 5 years. Clinical oral implants research, 15(6), 667-676. https://doi.org/10.1111/j.16000501.2004.01120.x 
Pye, A. D., Lockhart, D. E., Dawson, M. P., Murray, C. A., \& Smith, A. J. (2009). A review of dental implants and infection. The Journal of hospital infection, 72(2), 104-110. https://doi.org/10.1016/j.jhin.2009.02.010

Quirynen, M., De Soete, M., \& van Steenberghe, D. (2002). Infectious risks for oral implants: a review of the literature. Clinical oral implants research, 13(1), 1-19. https://doi.org/10.1034/j.1600-0501.2002.130101.x

Radaelli, M., Federizzi, L., Nascimento, G. G., Leite, F., \& Boscato, N. (2020). Early-predictors of marginal bone loss around morse taper connection implants loaded with single crowns: A prospective longitudinal study. Journal of periodontal research, 55(2), 174-181. https://doi.org/10.1111/jre.12699

Romanos, G. E., Javed, F., Delgado-Ruiz, R. A., \& Calvo-Guirado, J. L. (2015). Peri-implant diseases: a review of treatment interventions. Dental clinics of North America, 59(1), 157-178. https://doi.org/10.1016/j.cden.2014.08.002

Sailer, I., Mühlemann, S., Zwahlen, M., Hämmerle, C. H., \& Schneider, D. (2012). Cemented and screw-retained implant reconstructions: a systematic review of the survival and complication rates. Clinical oral implants research, 23 Suppl 6, 163-201. https://doi.org/10.1111/j.1600-0501.2012.02538.x

Sakka, S., Baroudi, K., \& Nassani, M. Z. (2012). Factors associated with early and late failure of dental implants. Journal of investigative and clinical dentistry, 3(4), 258-261. https://doi.org/10.1111/j.2041-1626.2012.00162.x

Shadid, R., \& Sadaqa, N. (2012). A comparison between screw- and cement-retained implant prostheses. A literature review. The Journal of oral implantology, 38(3), 298-307. https://doi.org/10.1563/AAID-JOI-D-10-00146

Sherif, S., Susarla, S. M., Hwang, J. W., Weber, H. P., \& Wright, R. F. (2011). Clinician- and patient-reported long-term evaluation of screw- and cementretained implant restorations: a 5-year prospective study. Clinical oral investigations, 15(6), 993-999. https://doi.org/10.1007/s00784-010-0460-4.

Silva, G. C., Cornacchia, T. M., de Magalhães, C. S., Bueno, A. C., \& Moreira, A. N. (2014). Biomechanical evaluation of screw- and cement-retained implant-supported prostheses: a nonlinear finite element analysis. The Journal of prosthetic dentistry, 112(6), 1479-1488. https://doi.org/10.1016/j.prosdent.2014.06.010

Ueda, T., Kremer, U., Katsoulis, J., \& Mericske-Stern, R. (2011). Long-term results of mandibular implants supporting an overdenture: implant survival, failures, and crestal bone level changes. The International journal of oral \& maxillofacial implants, 26(2), 365-372.

Vigolo, P., Mutinelli, S., Givani, A., \& Stellini, E. (2012). Cemented versus screw-retained implant-supported single-tooth crowns: a 10-year randomised controlled trial. European journal of oral implantology, 5(4), 355-364.

Wadhwani, C., Rapoport, D., La Rosa, S., Hess, T., \& Kretschmar, S. (2012). Radiographic detection and characteristic patterns of residual excess cement associated with cement-retained implant restorations: a clinical report. The Journal of prosthetic dentistry, 107(3), 151-157. https://doi.org/10.1016/S0022$3913(12) 60046-8$

Wadhwani, C., Rapoport, D., La Rosa, S., Hess, T., \& Kretschmar, S. (2012). Radiographic detection and characteristic patterns of residual excess cement associated with cement-retained implant restorations: a clinical report. The Journal of prosthetic dentistry, 107(3), 151-157. https://doi.org/10.1016/S00223913(12)60046-8 